\title{
Correction to: Dietary folate intake and metabolic syndrome in participants of PREDIMED-Plus study: a cross-sectional study
}

Eva-Maria Navarrete-Muñoz ${ }^{1}$. Jesus Vioque ${ }^{2,3,34}$ - Estefanía Toledo ${ }^{4,5} \cdot$ Alejando Oncina-Canovas $^{3}$. Miguel Ángel Martínez-González ${ }^{4,5,6}$. Jordi Salas-Salvadóo,7,8,9 . Dolores Corella ${ }^{4,10}$. Montserrat Fitó ${ }^{4,11}$. Dora Romaguera ${ }^{4,12}$. Ángel M. Alonso-Gómez ${ }^{4,13}$. Julia Wärnberg ${ }^{4,14}$. J. Alfredo Martínez ${ }^{4,15}$. Luís Serra-Majem ${ }^{4,16} \cdot$ Ramon Estruch $^{4,17}$. Francisco J. Tinahones ${ }^{4,18}$. José Lapetra ${ }^{4,19} \cdot$ Xavier Pintó ${ }^{4,20}$. Josep A. Tur ${ }^{4,12,21}$. José López-Miranda ${ }^{4,22}$. Aurora Bueno-Cavanillas ${ }^{2,23}$ • Pilar Matía-Martín ${ }^{24}$ - Lidia Daimiel ${ }^{25}$. Vicente Martín Sánchez ${ }^{2,26}$. Josep Vidal ${ }^{27,28}$. Ana Isabel de Cos Blanco ${ }^{4,29} \cdot$ Emili Ros $^{4,30}$ • Javier Diez-Espino ${ }^{4,5,31}$. Nancy Babio ${ }^{4,7,8,9} \cdot$ Rebeca Fernandez-Carrion 4,10 . Olga Castañer ${ }^{4,11}$. Antoni Colom ${ }^{12}$. Laura Compañ-Gabucio ${ }^{3}$. Itziar Salaverria Lete ${ }^{13}$. Edelys Crespo-Oliva ${ }^{4,14} \cdot$ Itziar Abete $^{4,15} \cdot$ Laura Tomaino 4,16,32 $_{\text {Rosa Casas }}$ Ran $^{417}$ José Carlos Fernandez-Garcia, ${ }^{418}$. José Manuel Santos-Lozano ${ }^{4,19} \cdot$ Iziar Sarasa $^{20}$ • José M. Gámez ${ }^{4,21,35,36}$. José M. Antonio Garcia-Rios ${ }^{4,22}$. Sandra Martín-Pelaez ${ }^{23} \cdot$ Miguel Ruiz-Canela $^{4,5}$ • Andrés Díaz-López ${ }^{4,7,8,9}$. Raul Martinez-Lacruz ${ }^{4,10} \cdot$ Maria Dolors Zomeño $^{4,11}$. Elena Rayó ${ }^{12} \cdot$ Cristina Gisbert Sellés $^{33} \cdot$ Silvia Canudas ${ }^{4,7,8,9}$. Albert Goday $^{4,11}$ - Manoli García-de-la-Hera ${ }^{2,3}$

Published online: 5 December 2020

๑) Springer-Verlag GmbH Germany, part of Springer Nature 2020

Correction to: European Journal of Nutrition https://doi.org/10.1007/s00394-020-02364-4

The original version of this article unfortunately contained a mistake. José M. Gámez was not listed among the authors.

Missing author, affiliation and order of the authors should be:

José M. Gámez ${ }^{4,21,35,36}$

${ }^{4}$ Centro de Investigación Biomédica en Red Fisiopatología de la Obesidad y la Nutrición (CIBEROBN), Institute of Health Carlos III, Madrid, Spain
${ }^{21}$ Research Group on Community Nutrition and Oxidative Stress, University of Balearic Islands, Palma de Mallorca, Spain

${ }^{35}$ Health Research Institute of the Balearic Islands (IdISBa), Palma de Mallorca, Spain

${ }^{36}$ Department of Cardiology, Hospital Son Llàtzer, 07198 Palma de Mallorca, Spain

The original article has been corrected.

The original article can be found online at https://doi.org/10.1007/ s00394-020-02364-4.

Extended author information available on the last page of the article 


\section{Affiliations}

Eva-Maria Navarrete-Muñoz ${ }^{1}$. Jesus Vioque ${ }^{2,3,34}$ - Estefanía Toledo ${ }^{4,5} \cdot$ Alejando Oncina-Canovas $^{3}$. Miguel Ángel Martínez-González ${ }^{4,5,6}$. Jordi Salas-Salvadóo, 4,8,9 . Dolores Corella ${ }^{4,10}$. Montserrat Fitó ${ }^{4,11}$. Dora Romaguera $^{4,12}$. Ángel M. Alonso-Gómez ${ }^{4,13}$. Julia Wärnberg ${ }^{4,14}$. J. Alfredo Martínez ${ }^{4,15}$. Luís Serra-Majem $^{4,16}$. Ramon Estruch ${ }^{4,17}$. Francisco J. Tinahones ${ }^{4,18}$. José Lapetra ${ }^{4,19}$ - Xavier Pintóo, 4,20 . Josep A. Tur ${ }^{4,12,21}$. José López-Miranda ${ }^{4,22}$. Aurora Bueno-Cavanillas ${ }^{2,23}$. Pilar Matía-Martín ${ }^{24}$. Lidia Daimiel25. Vicente Martín Sánchez ${ }^{2,26}$. Josep Vidal ${ }^{27,28}$. Ana Isabel de Cos Blanco ${ }^{4,29}$ - Emili Ros ${ }^{4,30}$. Javier Diez-Espino ${ }^{4,5,31}$. Nancy Babio ${ }^{4,7,8,9} \cdot$ Rebeca Fernandez-Carrion ${ }^{4,10}$. Olga Castañer ${ }^{4,11}$. Antoni Colom ${ }^{12}$ - Laura Compañ-Gabucio ${ }^{3}$. Itziar Salaverria Lete ${ }^{13} \cdot$ Edelys Crespo-Oliva $^{4,14} \cdot$ Itziar Abete $^{4,15} \cdot$ Laura Tomaino $^{4,16,32} \cdot$. Rosa Casas 4,17 . José Carlos Fernandez-Garcia, ${ }^{4,18}$. José Manuel Santos-Lozano ${ }^{4,19}$. Iziar Sarasa ${ }^{20}$. José M. Gámez ${ }^{4,21,35,36}$. José M. Antonio Garcia-Rios ${ }^{4,22}$. Sandra Martín-Pelaez ${ }^{23} \cdot$ Miguel Ruiz-Canela $^{4,5} \cdot$ Andrés Díaz-López $^{4,7,8,9}$. Raul Martinez-Lacruz ${ }^{4,10}$. Maria Dolors Zomeño ${ }^{4,11} \cdot$ Elena Rayó $^{12} \cdot$ Cristina Gisbert Sellés $^{33} \cdot$ Silvia Canudas $^{4,7,8,9}$. Albert Goday $^{4,11} \cdot$ Manoli García-de-la-Hera ${ }^{2,3}$

Jesus Vioque

vioque@umh.es

1 Grupo de Investigación en Terapia Ocupacional (InTeO), Department of Surgery and Pathology, Miguel Hernández University, 03550 Alicante, Spain

2 CIBER de Epidemiología y Salud Pública (CIBERESP), Institute of Health Carlos III, Madrid, Spain

3 Nutritional Epidemiology Unit, Universidad Miguel Hernández, ISABIAL-UMH, Alicante, Spain

4 Centro de Investigación Biomédica en Red Fisiopatología de la Obesidad y la Nutrición (CIBEROBN), Institute of Health Carlos III, Madrid, Spain

5 Department of Preventive Medicine and Public Health, University of Navarra, IdiSNA, Pamplona, Spain

6 Department of Nutrition, Harvard T.H. Chan School of Public Health, Boston, MA, USA

7 Departament de Bioquímica i Biotecnologia, Unitat de Nutrició, Universitat Rovira i Virgili, Reus, Spain

8 Nutrition Unit, University Hospital of Sant Joan de Reus, Reus, Spain

9 Institut d'Investigació Sanitària Pere Virgili (IISPV), Reus, Spain

10 Department of Preventive Medicine, University of Valencia, Valencia, Spain

11 Unit of Cardiovascular Risk and Nutrition, Institut Hospital del Mar de Investigaciones Médicas Municipal d'Investigació Médica (IMIM), Barcelona, Spain

12 Health Research Institute of the Balearic Islands (IdISBa), University Hospital Son Espases, Palma de Mallorca, Spain

13 Bioaraba Health Research Institute, Osakidetza Basque Health Service, Araba University Hospital, University of the Basque Country UPV/EHU, Vitoria-Gasteiz, Spain

14 Department of Nursing, School of Health Sciences, University of Málaga-Institute of Biomedical Research in Malaga (IBIMA), Málaga, Spain

15 Department of Nutrition, Food Sciences, and Physiology, Center for Nutrition Research, University of Navarra, Pamplona, Spain
16 Research Institute of Biomedical and Health Sciences (IUIBS), University of Las Palmas de Gran Canaria and Centro Hospitalario Universitario Insular Materno Infantil (CHUIMI), Canarian Health Service, Las Palmas de Gran Canaria, Spain

17 Department of Internal Medicine, Institut d'Investigacions Biomèdiques August Pi Sunyer (IDIBAPS), Hospital Clinic, University of Barcelona, Barcelona, Spain

18 Department of Endocrinology, Instituto de Investigación Biomédica de Málaga (IBIMA), Virgen de la Victoria Hospital, University of Málaga, Málaga, Spain

19 Department of Family Medicine, Research Unit, Distrito Sanitario Atención Primaria Sevilla, Sevilla, Spain

20 Lipids and Vascular Risk Unit, Internal Medicine, Hospital Universitario de Bellvitge, Hospitalet de Llobregat, Barcelona, Spain

21 Research Group on Community Nutrition and Oxidative Stress, University of Balearic Islands, Palma de Mallorca, Spain

22 Department of Internal Medicine, Maimonides Biomedical Research Institute of Cordoba (IMIBIC), Reina Sofia University Hospital, University of Cordoba, Cordoba, Spain

23 Department of Preventive Medicine and Public Health, University of Granada, Granada, Spain

24 Department of Endocrinology and Nutrition, Instituto de Investigación Sanitaria Hospital Clínico San Carlos (IdISSC), Madrid, Spain

25 Nutritional Control of the Epigenom Group, Precision Nutrition and Obesity Program, IMDEA Food, CEI UAM + CSIC, Madrid, Spain

26 Institute of Biomedicine (IBIOMED), University of León, León, Spain

27 CIBER Diabetes y Enfermedades Metabólicas (CIBERDEM), Instituto de Salud Carlos III (ISCIII), Madrid, Spain

28 Department of Endocrinology, Institut d'Investigacions Biomédiques August Pi Sunyer (IDIBAPS), Hospital Clinic, University of Barcelona, Barcelona, Spain 
29 Department of Endocrinology and Nutrition, Hospital Fundación Jimenez Díaz, Instituto de Investigaciones Biomédicas IISFJD, University Autonoma, Madrid, Spain

30 Lipid Clinic, Department of Endocrinology and Nutrition, Institut d'Investigacions Biomèdiques August Pi Sunyer (IDIBAPS), Hospital Clínic, Barcelona, Spain

31 Gerencia de Atención Primaria, Servicio Navarro de Salud-Osasunbidea, Pamplona, Spain

\author{
Department of Clinical and Community Health (DISCCO), \\ Università degli Studi di Milano, Milan, Italy
}

Centro Salud San Vicente del Raspeig, Alicante, Spain

34 Departamento Salud Pública, Campus San Juan, Universidad Miguel Hernández, Ctra. Nacional 332 s/n, 03550 Sant Joan d'Alacant, Spain

35 Health Research Institute of the Balearic Islands (IdISBa), Palma de Mallorca, Spain

36 Department of Cardiology, Hospital Son Llàtzer, 07198 Palma de Mallorca, Spain 